\title{
USO DE PRÉ, PRÓ E SIMBIÓTICOS COMO COADJUVANTES NO TRATAMENTO DO CÂNCER COLORRETAL
}

\author{
USE OF PRE-, PRO- AND SYMBIOTICS AS ADJUVANTS TO THE \\ TREATMENT OF COLORECTAL CANCER
}

\author{
Jésica Tamara Jacoby 1,2,3, Simone Guzzon ${ }^{4}$, \\ Luiz Fernando Wurdig Roesch ${ }^{5}$, Roberta Hack Mendes ${ }^{1}$
}

Clin Biomed Res. 2017;37(3):232-246

1 Universidade Regional Integrada do Alto Uruguai e das Missões (URI). Frederico Westphalen, RS, Brasil.

2 Hospital Regional de Palmitos. Palmitos, $\mathrm{SC}$, Brasil.

3 Hospital Padre João Berthier. São Carlos, SC, Brasil.

4 Universidade Comunitária da Região de Chapecó (UNOCHAPECÓ). Chapecó, SC, Brasil.

5 Centro Interdisciplinar de Pesquisas em Biotecnologia (CIP-BIOTEC), Universidade Federal do Pampa (UNIPAMPA). São Gabriel, RS, Brasil.

Autor correspondente: Roberta Hack Mendes betamendeshack@gmail.com Universidade Regional Integrada do Alto Uruguai e das Missões (URI) Rua Assis Brasil, 709, Itapajé. 98400-000, Frederico Westphalen, RS, Brasil.

\section{RESUMO}

Introdução: Definido como uma proliferação descontrolada de células malignas, o câncer colorretal (CCR) é um dos tumores malignos mais comuns, e a terceira causa de mortes relacionadas ao câncer. Várias estratégias têm sido estudadas para auxiliar na prevenção e no tratamento coadjuvante dos sintomas do CCR, entre elas a ingestão de probióticos, prebióticos ou simbióticos. Probióticos são microrganismos vivos, que quando administrados em quantidade adequada afetam beneficamente $o$ hospedeiro. Os probióticos são comumente encontrados em alimentos fermentados como em iogurtes por exemplo, ou na forma de suplementos que contém culturas microbianas vivas. Prebióticos são componentes alimentares não digeríveis que afetam beneficamente o hospedeiro, estimulando seletivamente a multiplicação ou atividade de populações microbianas desejáveis no cólon. Já, os simbióticos consistem na associação de probióticos e prebióticos. O objetivo do presente trabalho foi definir a validade do uso dos probióticos, prebióticos ou simbióticos como coadjuvantes no tratamento do CCR por meio de uma revisão sistemática da literatura.

Métodos: Foi realizada uma pesquisa nas bases de dados PUBMED, SCIELO, COCHRANE e CLINICAL TRIALS. Os termos de busca foram: "colorectal cancer AND probiotics", "colorectal cancer AND prebiotics".

Resultados: Dos 68 artigos elegíveis, 14 foram revisados, com publicação entre 2012 e 2017, escritos no idioma inglês, português ou espanhol. O número amostral variou de 38 a 310 pacientes, com idade entre 18 e 75 anos, a duração do tratamento foi de 3 dias a 3 meses. Em 3 dos estudos foram utilizados simbióticos, em 1 prebióticos, e em 10 probióticos. As cepas de probióticos continham entre 1 e 10 substâncias, prebióticos 4 substâncias e os simbióticos entre 5 e 8.

Conclusão: $O$ trabalho possibilitou o reconhecimento dos principais microrganismos que vem sendo estudados no tratamento concomitante do CCR. A maioria dos estudos analisados mostrou efeitos benéficos na diminuição da proteína $C$ reativa, da incidência e severidade da diarreia, risco de complicações pós-operatórias como sepse, ventilação mecânica e vazamento da anastomose, além de propiciar uma recuperação da função intestinal mais rápida. A presente revisão sistemática ressalta a importância dos pré e probióticos concomitante aos tratamentos de CCR, porém o número limitado de artigos dificulta a generalização dos resultados obtidos, sendo necessários futuros estudos de longa duração para elucidar melhor esta relação.

Palavras-chave: Neoplasias colorretais; microbiota; terapias coadjuvantes

Introduction: Defined as an uncontrolled proliferation of malignant cells, colorectal cancer (CRC) is one of the most common malignancies and the third leading cause of cancer-related deaths. Several strategies have been studied to prevent CRC and/or work as an adjuvant treatment for CRC symptoms, including the use of probiotics, prebiotics or symbiotics. Probiotics are live microorganisms that when administered 
in adequate amounts result in health benefit to the host. They are commonly found in fermented foods such as yogurts or in the form of supplements that contain live microbial cultures. Prebiotics are nondigestible food components that beneficially affect the host by selectively stimulating the multiplication or activity of desirable microbial populations in the colon. Symbiotics consist of the association of probiotics and prebiotics. The aim of the present study was to define the effectiveness of the use of probiotics, prebiotics and symbiotics as adjuvants to the treatment of CRC by means of a systematic review of the literature.

Methods: A search was performed on the PubMed, SciELO, Cochrane and Clinical Trials databases. The search terms were "colorectal cancer AND probiotics", "colorectal cancer AND prebiotics".

Results: Of the 68 potentially eligible articles, 14 were revised, published between 2012 and 2017, and written in English, Portuguese or Spanish. The sample size of these studies ranged from 38 to 310 patients, aged between 18 and 75 years. The period of treatment ranged from 3 days to 3 months. Symbiotics were used in three studies, prebiotics were used in one study, and probiotics were used in 10 studies. Probiotic strains contained between one and 10 substances, prebiotics contained four substances, and symbiotics contained between five and eight substances.

Conclusions: This study allowed the recognition of the main microorganisms that have been studied in the concomitant treatment of CRC. Most of the studies analyzed showed beneficial effects on the reduction of C-reactive protein, the incidence and severity of diarrhea and the risk of postoperative complications such as sepsis, mechanical ventilation and leakage of anastomosis, in addition to providing a faster recovery of the intestinal function. The present systematic review emphasizes the importance of the use of pre- and probiotics concomitant with CRC treatments, but the limited number of articles makes it difficult to generalize the results obtained. Further long-term studies are needed to elucidate this relationship.

Keywords: Colorectal neoplasms; microbiota; adjuvant therapies

O câncer é definido como uma proliferação descontrolada de células malignas em um hospedeiro e considerado uma das principais causas de morte em todo o mundo ${ }^{1}$. Entre os tipos de câncer conhecidos, o câncer colorretal (CCR) é um dos tumores malignos mais comuns em homens e mulheres. A incidência mundial dessa doença é significativa, com cerca de um milhão de casos por ano. Além disso, é a causa de mais de 500.000 mortes por ano, sendo a terceira causa mais comum de mortes relacionadas ao câncer ${ }^{2}$. No Brasil, estima-se uma incidência de 34.280 novos casos ao ano e 15.415 óbitos $^{3}$.

O CCR possui múltiplas origens, tendo como principais fatores de risco hereditariedade, doenças inflamatórias, idade, dieta, obesidade e sedentarismo $\mathrm{o}^{4-6}$. Entre os fatores de risco ambientais, a dieta contribui com cerca de $35 \%$ dos novos casos. O alto consumo de calorias, gorduras e carnes vermelhas associado ao baixo consumo de frutas e hortaliças propicia uma maior incidência da doença. Acredita-se que uma dieta variada e adequada possa prevenir de três a quatro milhões de novos casos de câncer por ano ${ }^{6}$.

Além de os fatores genéticos e ambientais desempenharem um papel importante no desenvolvimento da doença, o envolvimento de comunidades microbianas também tem sido recentemente reconhecido e associado ao CCR ${ }^{1}$. Segundo Gao et al. ${ }^{5}$, uma das características comuns dos fatores de risco é que todos podem causar alterações na estrutura da microbiota intestinal, desencadeando o CCR.

Os tratamentos comuns para o CCR são cirurgias como a colostomia, que consiste na criação cirúrgica de uma abertura (ostomia) que se insere no cólon com a finalidade de eliminar as fezes, podendo ser temporária ou permanente. Também pode ter efeitos adversos ou complicações como sepse, infecções, ventilação mecânica, fuga anastomótica, entre outros. Além do tratamento cirúrgico, é realizada conjuntamente a quimio ou radioterapia, o que traz mais efeitos colaterais, como náuseas, êmese, diarreia e mucosite. Todos esses sintomas podem vir a afetar a condição nutricional, o equilíbrio hidroeletrolítico e a qualidade de vida do paciente ${ }^{7,8}$.

Várias estratégias para auxiliar na prevenção e no tratamento coadjuvante antineoplásico vêm sendo estudadas, entre elas a ingestão de probióticos, prebióticos ou simbióticos. Alguns estudos vêm demonstrando que o consumo de probióticos 
e/ou prebióticos pode ter efeito protetor contra o câncer, além de diminuir os sintomas da doença ${ }^{9}$. Os probióticos têm efeitos quimiopreventivos contra o câncer colorretal, produzindo agentes antitumorais ${ }^{9-12}$.

Probióticos são microrganismos vivos que, quando administrados em quantidade adequada, afetam beneficamente o hospedeiro ${ }^{6,13}$, enquanto os prebióticos são componentes alimentares não digeríveis que afetam beneficamente o hospedeiro, estimulando seletivamente a proliferação ou atividade de populações de bactérias desejáveis no cólon ${ }^{6}$. Prebióticos, como aqueles que são fonte de fibras, promovem aumento das concentrações de ácidos graxos de cadeia curta (AGCCs), os quais são formados através da fermentação por bactérias colônicas. Os AGCCs desempenham uma importante função no metabolismo intestinal, induzindo a diferenciação de linhagens celulares e inibindo o crescimento e desenvolvimento do epitélio hiperproliferativo, além de possuírem ação celular anticarcinogênica. Os AGCCs estão envolvidos no processo de controle da promoção e diferenciação celular, apoptose e supressão de células neoplásicas devido à ação do butirato, que induz esses efeitos através da regulação de vários oncogenes e indução de hiperacetilação de histonas ${ }^{14,15}$.

Já os simbióticos consistem na associação de probióticos e prebióticos. Alternativamente, esse efeito simbiótico pode ser direcionado às diferentes "regiões-alvo" do trato gastrintestinal, os intestinos delgado e grosso. O consumo de probióticos e de prebióticos selecionados apropriadamente pode aumentar os efeitos benéficos de cada um deles, uma vez que o estímulo de cepas probióticas conhecidas leva à escolha dos pares simbióticos substrato-microrganismo ideais ${ }^{6}$.

Segundo Machado et al. ${ }^{9}$, existe uma relação entre indivíduos que utilizam simbióticos e uma proteção contra o aparecimento de CCR, porém não existem comprovações bem estabelecidas na literatura até o momento. Evidências demonstram que alterações físico-químicas do cólon e da microbiota intestinal causadas pelo consumo de simbióticos, como o aumento da produção de AGCC e a elevação de compostos antitumorais ou antimutagênicos, podem propiciar essa proteção contra o câncer, pois resultam em uma melhor resposta imune às alterações da microbiota.

É de extrema importância, portanto, o desenvolvimento de terapias coadjuvantes que possam auxiliar na diminuição do desconforto no quadro clínico do paciente com CCR. O uso de pro e prebióticos pode auxiliar os pacientes na diminuição dos sintomas, evitando infecções bacterianas, prevenindo e diminuindo a incidência e severidade da diarreia, recuperando mais rapidamente a função intestinal, evitando translocação bacteriana e melhorando a resposta ao estresse sistêmico e da imunidade sistêmica, além de diminuir complicações pós-cirúrgicas. Assim, o objetivo do presente trabalho foi definir a validade do uso dos probióticos, prebióticos ou simbióticos como coadjuvantes no tratamento do CCR por meio de uma revisão sistemática da literatura.

\section{MÉTODOS}

\section{Pesquisa da Literatura e Seleção de Estudos}

Neste trabalho, utilizou-se a revisão sistemática para identificar, selecionar e avaliar criticamente as pesquisas consideradas relevantes, resumindo evidências associadas à eficácia e segurança das intervenções de atenção à saúde. Pode apresentar resultados conflitantes e/ou coincidentes, ou, ainda, identificar temas que precisam de evidência antes de serem aplicados ${ }^{16,17}$. O protocolo de avaliação pré-definido foi registrado no International Prospective Register of Systematic Reviews (PROSPERO) sob número CRD42017068750. Foi realizada uma pesquisa da literatura nos seguintes bancos de dados eletrônicos: Scientific Electronic Library Online (SciELO), PubMed, Cochrane e Clinical Trials. As palavras-chave utilizadas foram "colorectal cancer AND probiotics", "colorectal cancer AND prebiotics". Como "symbiotics" representa a combinação de probióticos e prebióticos, esse termo não foi utilizado na busca.

A busca dos artigos nas bases de dados foi realizada por dois revisores independentes, sendo a revisão inicial realizada apenas com base nos títulos dos artigos e resumos. Depois disso, os artigos potencialmente elegíveis foram avaliados. Como critérios de inclusão foram considerados: estudo clínico, aleatorizado ou não, realizado com pacientes adultos e/ou idosos de ambos os sexos, com idade entre 18 e 75 anos, com uso de probióticos e/ou prebióticos, diagnóstico de câncer colorretal e em algum tipo de tratamento, com artigos disponíveis nas línguas português, inglês e espanhol, publicados nos últimos 5 anos, entre 2012 e 2017. Foram excluídos artigos de revisão, revisões sistemáticas, metanálises, resumos de congressos e estudos experimentais com animais e crianças. O presente estudo baseou-se na Declaração dos Relatórios Preferenciais para Revisão Sistemática e Metanálise (PRISMA) ${ }^{18}$.

\section{Qualidade dos Estudos Selecionados}

O risco de viés para cada ensaio clínico aleatorizado (ECA) incluído no estudo foi avaliado utilizando a ferramenta da Cochrane Collaboration ${ }^{19}$ para determinar o risco de viés em um desfecho único, com relação a todos os estudos. Para avaliação dos estudos longitudinais e caso-controle, foi utilizada o checklist ECA conforme apresentado na Tabela 1. O fluxograma com a seleção de artigos é apresentado na Figura 1. 


\section{RESULTADOS}

A busca bibliográfica nas bases de dados resultou em 68 artigos (33 na PubMed, três no SciELO, 14 no Clinical Trials e 18 no Cochrane). De acordo com os critérios de inclusão, foram selecionados 14 artigos originais para revisão no presente estudo. Conforme pode-se observar na Tabela 2. Foram excluídos oito artigos com base no idioma,

Tabela 1: Avaliação de risco de viés para ensaios clínicos aleatorizados (ECAs) (Cochrane Collaboration).

\begin{tabular}{|c|c|c|c|c|c|c|}
\hline Estudo & $\begin{array}{c}\text { Geração da } \\
\text { randomização }\end{array}$ & Alocação & $\begin{array}{l}\text { Cegamento de } \\
\text { participantes/ } \\
\text { pesquisadores }\end{array}$ & $\begin{array}{l}\text { Cegamento } \\
\text { de desfecho }\end{array}$ & $\begin{array}{c}\text { Descrição } \\
\text { de } \\
\text { perdas e } \\
\text { exclusões }\end{array}$ & $\begin{array}{l}\text { Intenção } \\
\text { de tratar }\end{array}$ \\
\hline Zhang et al. ${ }^{20}$ & $\downarrow$ & $\downarrow$ & $\downarrow$ & $\downarrow$ & $\downarrow$ & $\downarrow$ \\
\hline Stephens \& Hewett ${ }^{21}$ & $\downarrow$ & $\downarrow$ & $\downarrow$ & $\downarrow$ & $\downarrow$ & $\downarrow$ \\
\hline Liu et al. ${ }^{22}$ & $\downarrow$ & $\downarrow$ & $\downarrow$ & $\downarrow$ & $\downarrow$ & $\downarrow$ \\
\hline Lee et al..$^{10}$ & $\downarrow$ & $\downarrow$ & $\downarrow$ & $\downarrow$ & $\downarrow$ & $\downarrow$ \\
\hline Sadahiro et al. ${ }^{23}$ & $\downarrow$ & $?$ & $\downarrow$ & $\downarrow$ & $\downarrow$ & $\downarrow$ \\
\hline Gao et al..$^{5}$ & $\downarrow$ & $\downarrow$ & $\downarrow$ & $\downarrow$ & $\downarrow$ & $\downarrow$ \\
\hline Mego et al. ${ }^{24}$ & $\downarrow$ & $\downarrow$ & $\downarrow$ & $\downarrow$ & $\downarrow$ & $\downarrow$ \\
\hline Kotzampassi et al. ${ }^{25}$ & $\downarrow$ & $\downarrow$ & $\downarrow$ & $\downarrow$ & $\downarrow$ & $\downarrow$ \\
\hline Kotzampassi et al. ${ }^{26}$ & $\downarrow$ & $\downarrow$ & $\downarrow$ & $\downarrow$ & $\downarrow$ & $\downarrow$ \\
\hline Scartoni et al. ${ }^{27}$ & $?$ & $?$ & $?$ & $\downarrow$ & $\downarrow$ & $\downarrow$ \\
\hline Liu et al. ${ }^{28}$ & $\downarrow$ & $\downarrow$ & $\downarrow$ & $\downarrow$ & $\downarrow$ & $\downarrow$ \\
\hline Krebs $^{29}$ & $\downarrow$ & $\downarrow$ & $\downarrow$ & $\downarrow$ & $\downarrow$ & $\downarrow$ \\
\hline Yang et al..$^{30}$ & $\downarrow$ & $\downarrow$ & $\downarrow$ & $\downarrow$ & $\downarrow$ & $\downarrow$ \\
\hline Theodoropoulos et al. ${ }^{8}$ & 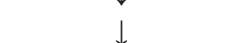 & 西 & 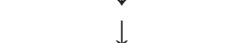 & 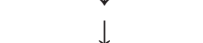 & 1 & 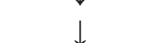 \\
\hline
\end{tabular}

Legenda: $\downarrow$ Sim/Baixo risco | $\uparrow$ Não/Alto risco | ? Não é claro.

68 artigos encontrados

(SciELO/PubMed/Cochrane/Clinical

Trials)

Artigos excluídos com base no

idioma

$(\mathrm{n}=8)$

Estudos incluídos

$(\mathrm{n}=60)$

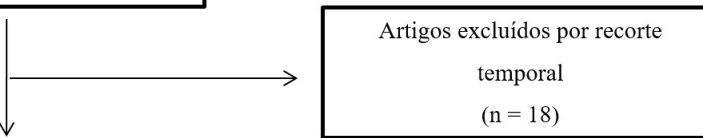

Estudos incluídos

$(n=42)$

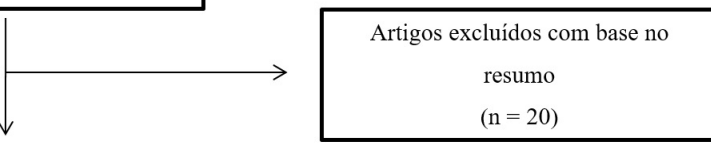

Estudos incluídos

$(\mathrm{n}=22)$

Artigos sem resultados publicados

$(\mathrm{n}=8)$

Estudos incluídos

$(\mathrm{n}=14)$

Figura 1: Fluxograma do resultado de busca. 


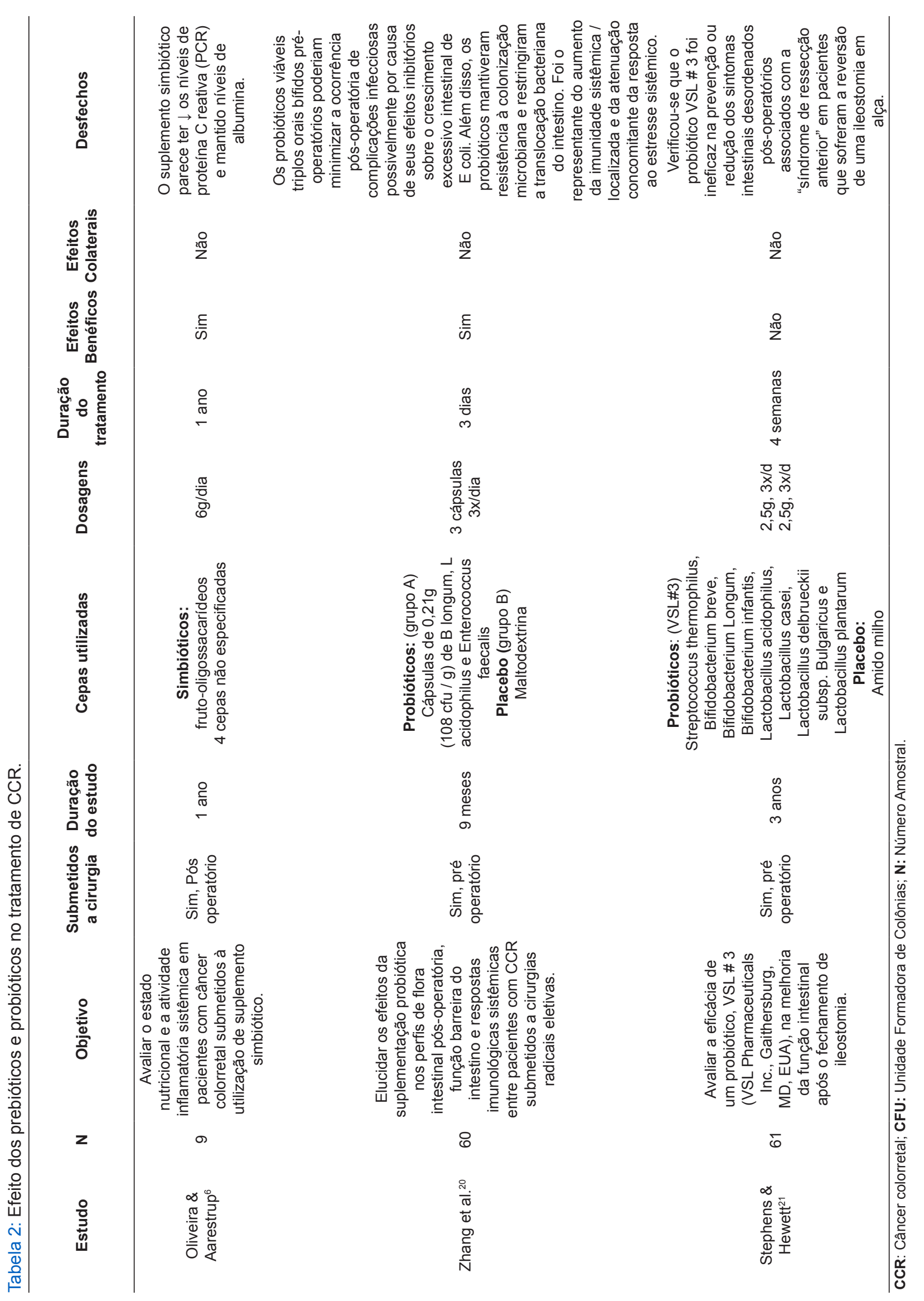




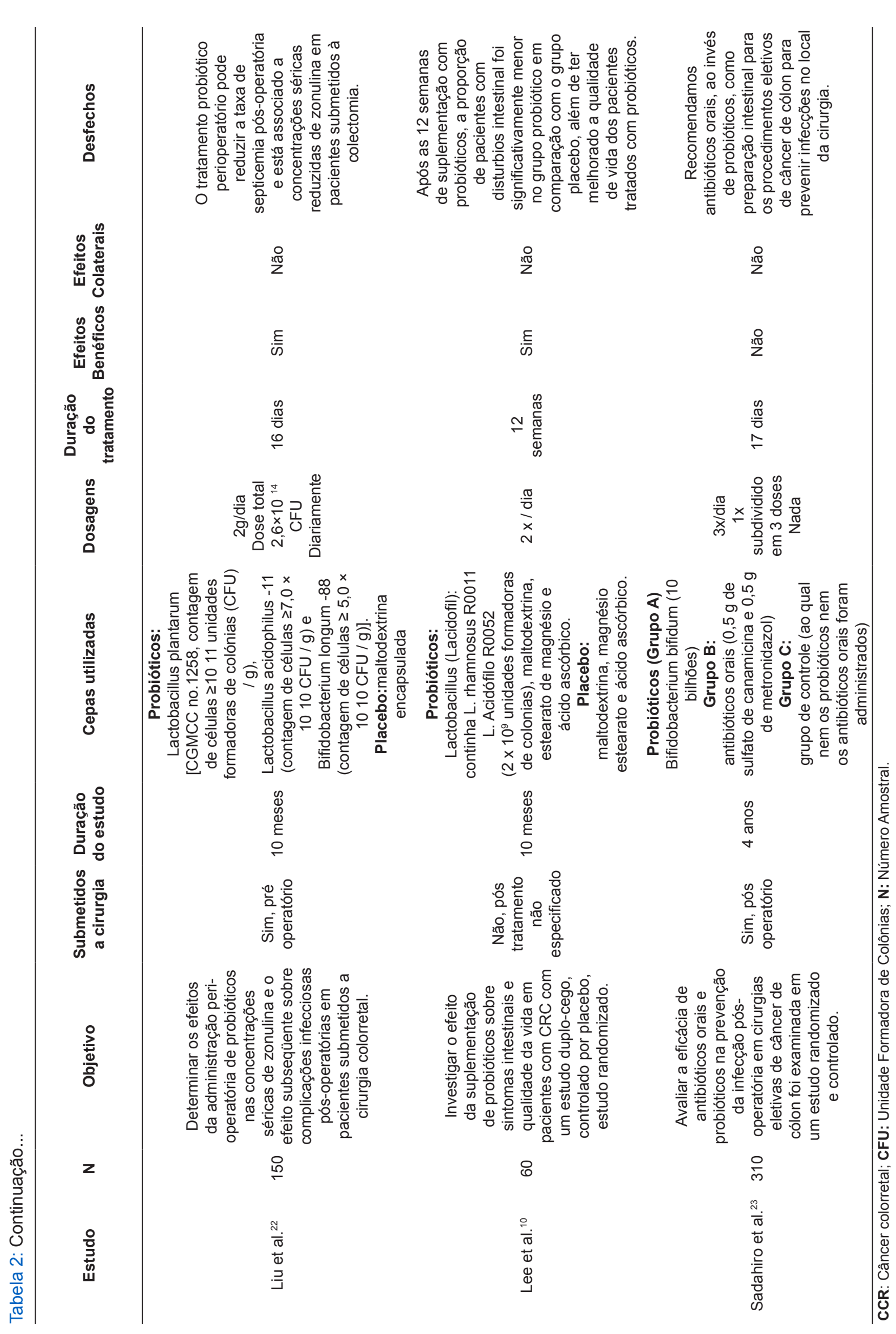




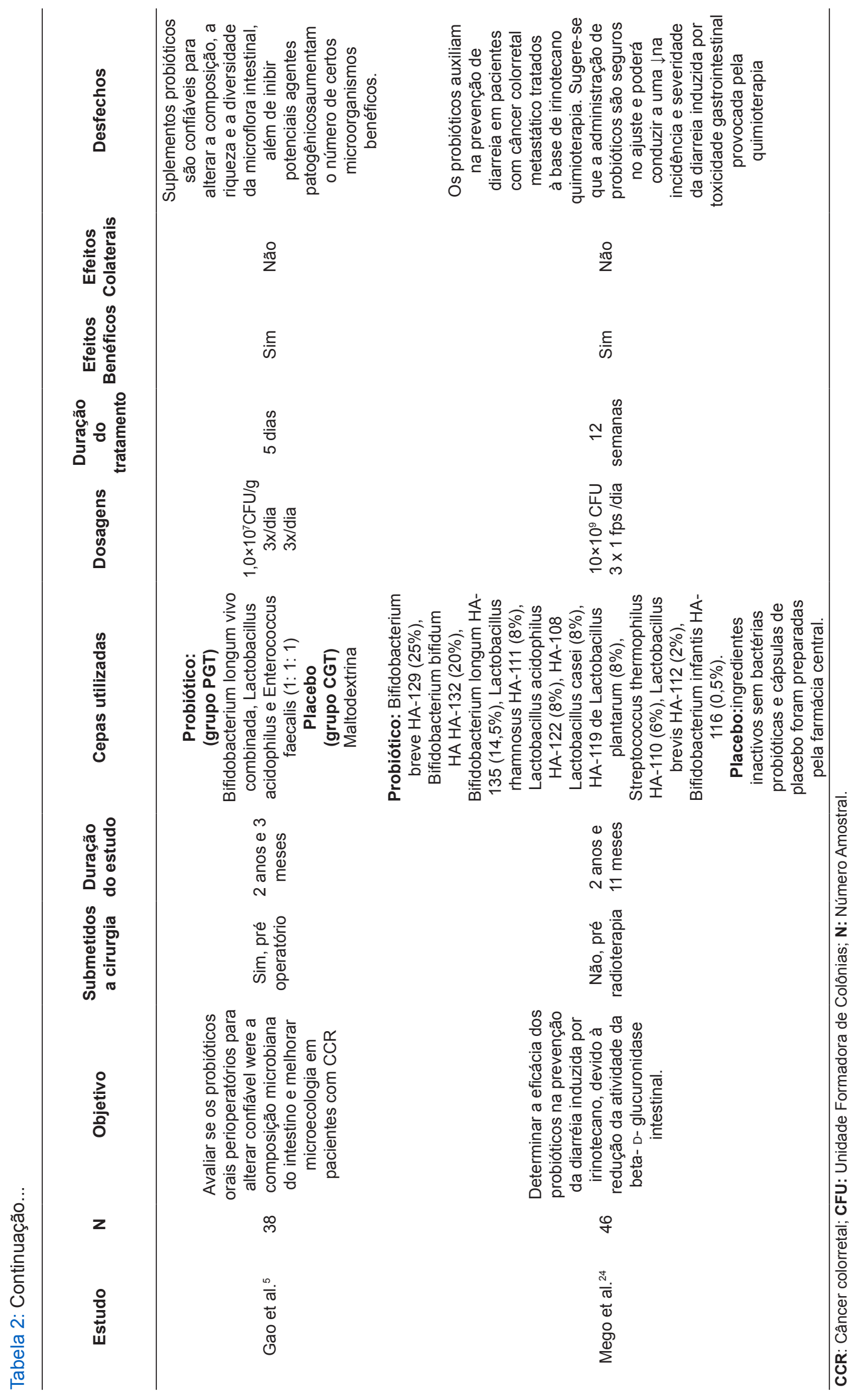




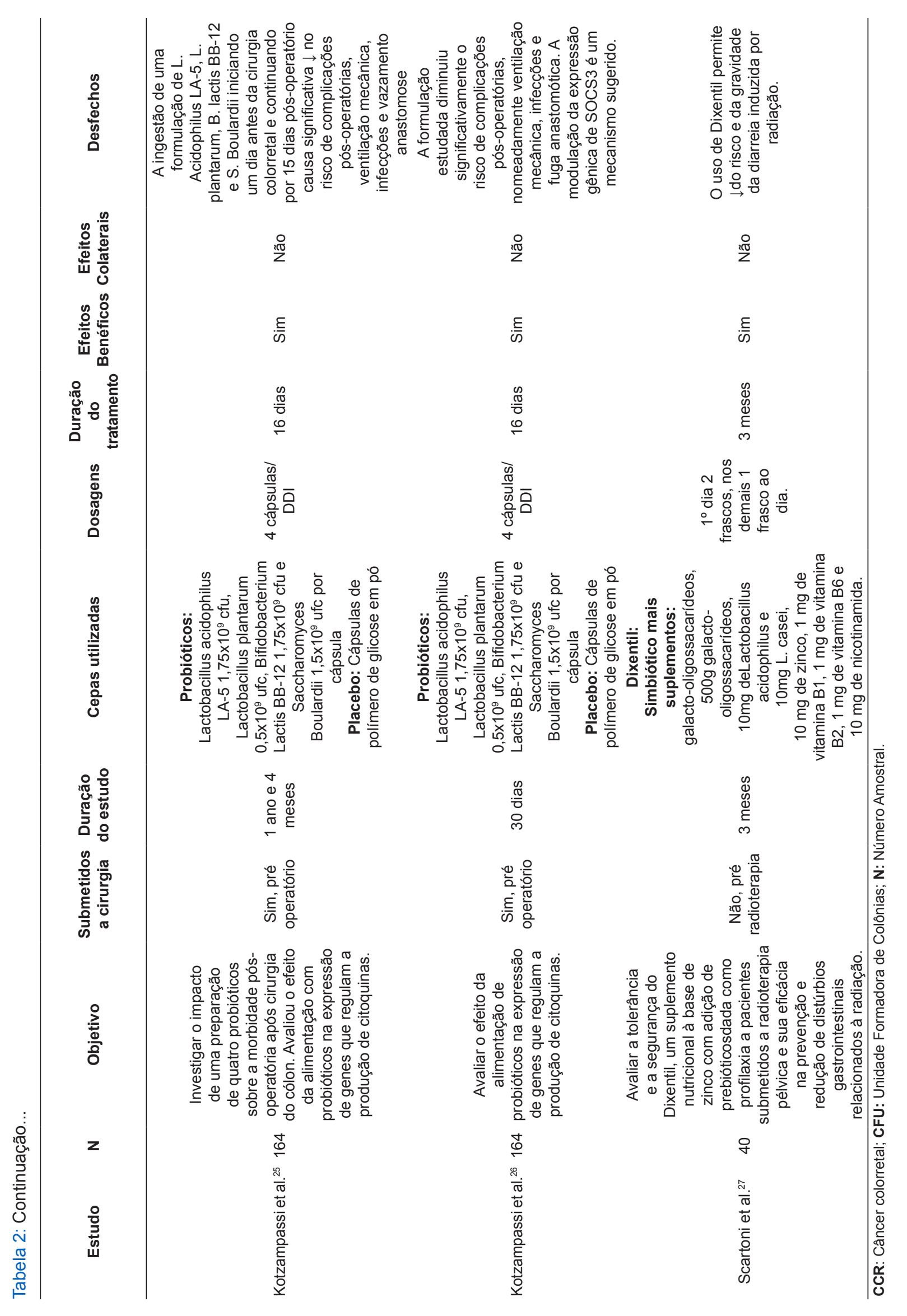




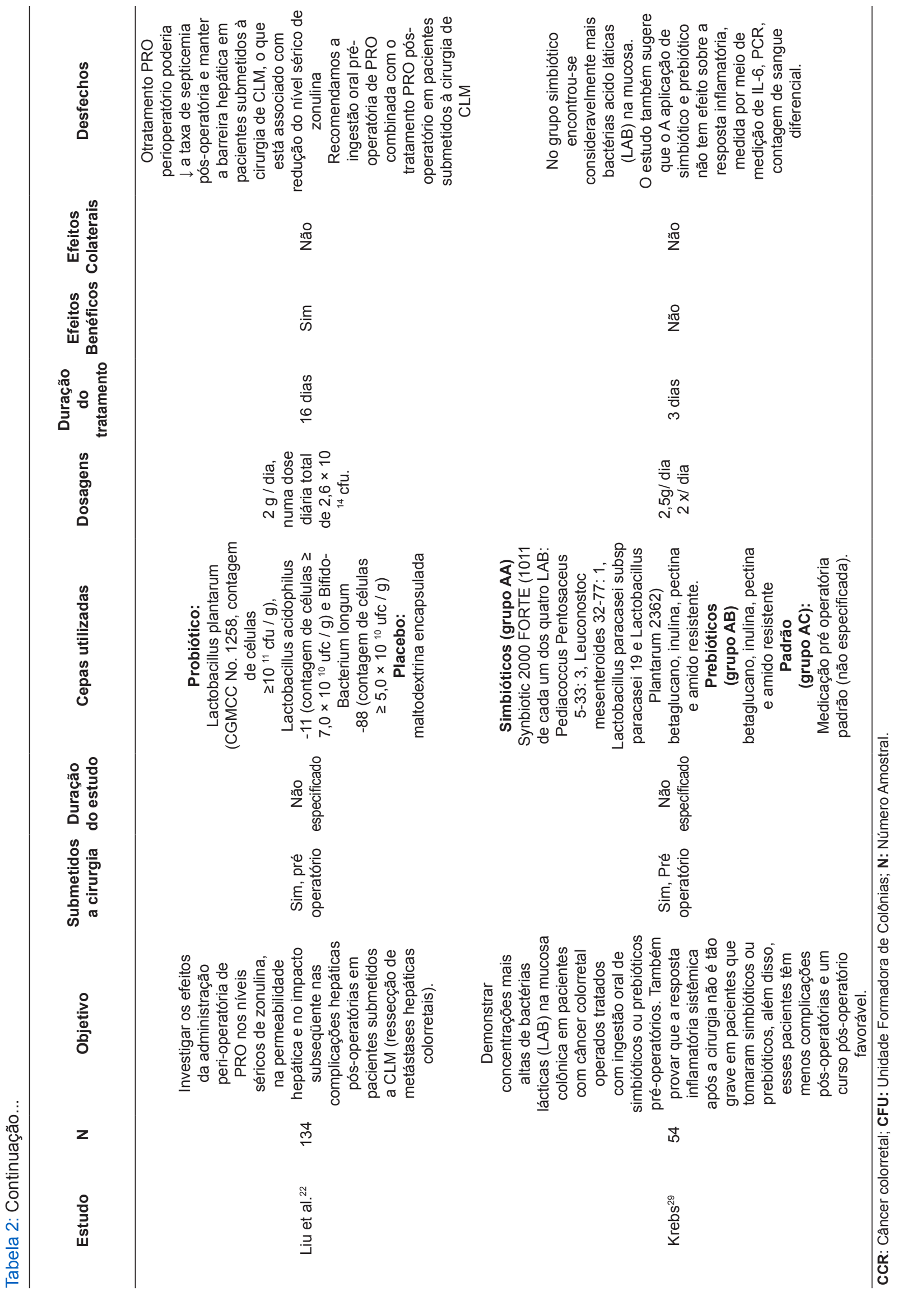




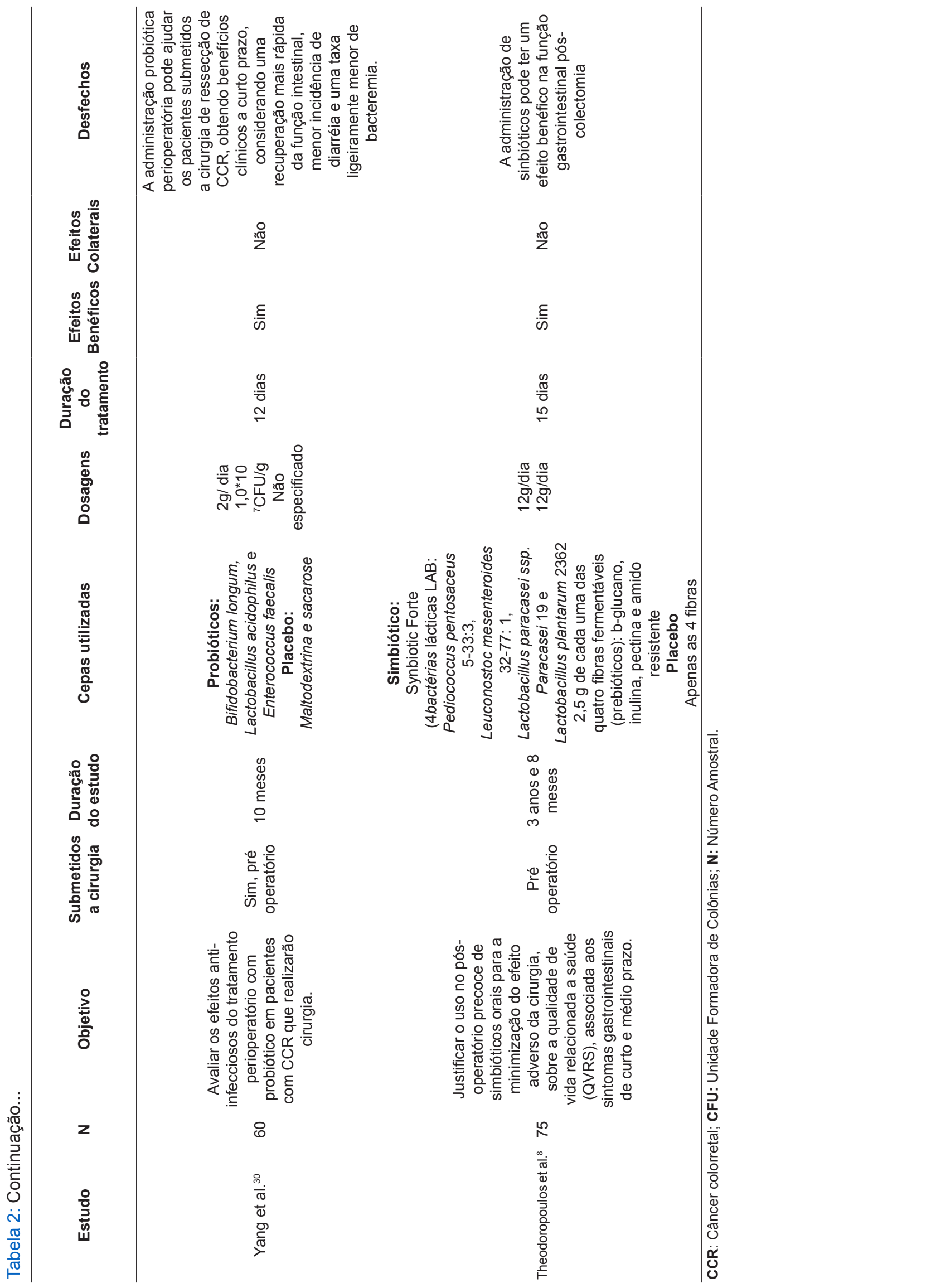


18 pela data de publicação anterior ao ano de 2012 , 20 com base no resumo e oito por não apresentarem resultados até o presente momento.

A maioria dos estudos selecionados era do tipo ensaio clínico aleatorizado, controlado por placebo, duplo-cego e escrito no idioma inglês. O número amostral variou de 38 a 310 pacientes em tratamento de CCR, com duração de 3 meses a 4 anos de estudo, sendo que em dois estudos não fica claro o tempo de duração do tratamento de CCR. A variação da duração da terapia com pre, pro ou simbióticos nos estudos foi de 3 dias até 3 meses.

Em três estudos, foram utilizados simbióticos, sendo que um deles também usava prebióticos em um dos grupos, e, em 11 estudos, foram utilizados probióticos. As cepas de probióticos continham entre uma e 10 substâncias; as de prebióticos, quatro substâncias; e as de simbióticos, entre cinco e oito substâncias.

Como prebióticos (incluindo simbióticos), foram utilizados fruto-oligossacarídeos (1), galacto-oligossacarídeos (1) e betaglucano, inulina, pectina e amido resistente (3), sendo que um dos simbióticos também continha suplementação de zinco e vitaminas do complexo $B$.

Dos estudos que fizeram uso de probióticos, $86,7 \%(n=13)$ utilizaram Lactobacillus, entre eles L. Paracasei (4), L. Plantarum (8), L. Acidophilus (11), L. Rhamnosus (2), L. brevis (1) e L. delbrueckii (1). Bifidobacterium foi utilizado em $60 \%(n=9)$ dos estudos, entre eles $B$. lactis (2), B. longum (7), B. breve (2), $B$. bifidum (2) e $B$. infantis (2). Também foram usados Pediococcus pentosaceus (2), Leuconostoc mesenteroides (2), Saccharomyces boulardii (2), Enterococcus faecalis (3), Bulgaricus (1) e Streptococcus thermophilus (2).

Observou-se que 64,3\% ( $n=9)$ das intervenções do estudo ocorreram na fase pré-operatória, $14,3 \%$ $(n=2)$, na fase pós-operatória, $14,3 \%(n=2)$, na fase pré-radioterapia e $7,1 \%(n=1)$, em pacientes pós-tratamento não especificado.

Efeitos benéficos, como a diminuição da proteína C-reativa (PCR) e da incidência e severidade da diarreia e do risco de complicações pós-operatórias como sepse, ventilação mecânica e vazamento da anastomose, além da recuperação da função intestinal mais rápida, foram observados em $78,6 \%$ $(n=11)$ dos estudos. Em 21,4\% $(n=3)$, os resultados esperados não foram atingidos. Nenhum dos estudos apresentou efeitos colaterais pelo uso de probióticos, prebióticos ou simbióticos.

\section{DISCUSSÃO}

O uso de probióticos e prebióticos diminui o risco de complicações pós-operatórias, como septicemia ${ }^{22,28}$, ventilação mecânica, infecções, pneumonia e vazamento da anastomose ${ }^{25,26}$, além de trazer benefícios clínicos em curto prazo através da recuperação mais rápida da função intestinal, com menor incidência de diarreia e alta hospitalar mais rápida ${ }^{26,30}$. Além disso, o uso de probióticos e prebióticos auxilia na manutenção da resistência à colonização microbiana por microrganismos indesejáveis e restringe a translocação bacteriana no intestino. Observa-se também o aumento da imunidade sistêmica/localizada e da atenuação concomitante da resposta ao estresse sistêmico ${ }^{20}$. Suplementos probióticos são confiáveis para alterar a composição, a riqueza e a diversidade da microbiota intestinal. Além disso, inibem potenciais agentes patogênicos e aumentam o número de microrganismos benéficos ${ }^{5}$.

A utilização de probióticos no pós-operatório também foi descrita como benéfica por Oliveira ${ }^{12}$, que verificou a redução das concentrações de PCR e a manutenção dos níveis de albumina sérica após cirurgias de tratamento de CCR. O mecanismo exato que causa o aumento das concentrações séricas de PCR em pacientes com CCR não está claro. Um dos mecanismos propostos é o aumento do nível sérico das citocinas interleucina 6 (IL-6) e fator de necrose tumoral (TNF) em pacientes com câncer, o que estimularia o fígado a sintetizar a PCR. Desse modo, a PCR pode ser utilizada como um índice da função imune intestinal para o prognóstico de pacientes com $\mathrm{CCR}^{28}$.

Os níveis de IL-6 estão sempre relacionados aos estudos com pacientes oncológicos, pois a IL-6 é uma potente citocina que possui ação imunológica, mobilizando a produção de granulócitos e macrófagos no organismo ${ }^{31}$. A IL-6 está relacionada ao combate das reações inflamatórias nos pacientes com câncer. Dessa forma, uma contagem de IL-6 menor pode ter uma ação anti-inflamatória, e, consequentemente, o paciente apresentará um melhor prognóstico pós-cirúrgico ${ }^{32}$.

Gamallat et al.$^{33}$ observaram que os probióticos possuem uma atividade de quimioprevenção e um possível efeito protetor no desenvolvimento e progressão da carcinogênese do cólon. Essa atividade anticarcinogênica atribuída aos probióticos é descrita pela modificação da composição e alterações na atividade metabólica da microbiota intestinal, pela ligação e degradação de compostos carcinogênicos presentes no lúmen intestinal e pela produção de compostos com atividade anticarcinogênica, como os AGCCs e o ácido linoleico conjugado, além da imunomodulação, melhora da barreira intestinal, inibição da proliferação de células cancerosas e indução da apoptose ${ }^{34}$. 
Assim, a comunidade microbiana intestinal tem demonstrado influência no início e na progressão do CCR. A proteção desempenhada pelos probióticos baseia-se na hipótese de que a disbiose é a principal causa do CCR. Portanto, com a modificação da microbiota intestinal, pode-se prevenir o desenvolvimento do $\mathrm{CCR}^{34,35}$.

Com o intuito de avaliar essa proposição, Pala et al. ${ }^{36}$ avaliaram a correlação entre o consumo de iogurte e o risco de desenvolver CCR, descobrindo que o consumo de iogurte estava diretamente relacionado a um menor risco de CCR. Em estudo publicado por Sah et al. ${ }^{37}$, foi possível observar que algumas cepas probióticas presentes em iogurtes são confiáveis para produzir peptídeos antioxidantes, eliminando radicais livres, consequentemente reduzindo o estresse oxidativo no lúmen intestinal $e$, dessa forma, prevenindo ou retardando o aparecimento do CCR. Além de apontar que o tratamento com probióticos inibe a inflamação e a angiogênese no desenvolvimento do tumor, Gamallat et al. ${ }^{33}$ demonstraram, em um estudo experimental em modelo animal, que o tratamento com $1 \mathrm{~mL}$ ao dia de Lactobacillus rhamnosus ( $10^{9}$ unidades formadoras de colônia) reduziu significativamente a incidência, a multiplicidade e o volume do tumor se comparado ao grupo controle, que ingeriu placebo. Da mesma forma, Kuugbee et al.$^{38}$ descreveram em seu estudo, também experimental em modelo animal, que o tratamento com cepas probióticas (Lactobacillus acidophilus, Bifidobacteria bifidum e Bifidobacteria infantum) alterou a microbiota intestinal, o que foi associado a uma diminuição de incidência, volume tumoral e multiplicidade/contagem tumoral.

Corroborando os estudos de Kuugbee ${ }^{38} \mathrm{e}$ Gamallat et al. ${ }^{33}$, Li et al. ${ }^{39}$, em um estudo experimental com camundongos, observaram o potencial terapêutico dos probióticos, os quais retardaram significativamente o crescimento tumoral, reduzindo o tamanho e o peso do tumor em $40 \%$ em comparação com o grupo controle. Os probióticos modificaram o perfil bacteriano intestinal para bactérias benéficas, incluindo Prevotella e Oscillibacter, que são conhecidos produtores de metabólitos anti-inflamatórios, que posteriormente reduziram a polarização da Th17 e promoveram a diferenciação das células Treg/Tr1 anti-inflamatórias no intestino. Li et al ${ }^{39}$ também descreveram que a ingestão precoce de probiótico pode preparar o corpo com uma base anti-inflamatória e limitar a geração de excesso de células Th17 no intestino, o qual poderia ser recrutado para formação de tumores e inflamação em outros tecidos. Também foi observado aumento de AGCCs, produzidos por comunidades microbianas estimuladas pelo uso do probiótico. Os AGCCs têm apresentado efeitos anti-inflamatórios no intestino, regulando as citocinas pró-inflamatórias, induzindo a diferenciação das células T reguladoras e suprimindo a polarização da Th1 $17^{40}$. Também foi observado um efeito anticancerígeno das bactérias ácido-láticas, as quais foram descritas como redutoras da incidência e multiplicidade de tumores por Leu et al. ${ }^{41}$ Observou-se também que a combinação simbiótica de amido resistente e Bifidobacterium lactis protegeu significativamente contra o desenvolvimento de CCR em modelo animal.

Segundo Krebs ${ }^{29}$, Sadahiro et al. ${ }^{23}$ e Stephens \& Hewett ${ }^{21}$, o uso de pre, pro e/ou simbióticos no pré-operatório não parece apresentar efeitos benéficos. Não demonstra efeitos sobre as concentrações de IL-6, PCR e contagem diferencial de leucócitos ${ }^{29}$, sendo ineficaz na prevenção ou redução dos sintomas intestinais pós-operatórios associados à "síndrome de ressecção anterior" em pacientes que sofreram a reversão de uma ileostomia em alça ${ }^{21}$. Os pesquisadores ressaltaram que os antibióticos orais foram mais eficazes na prevenção da infecção pós-operatória em cirurgias eletivas de câncer de cólon do que os probióticos ${ }^{23}$.

Por outro lado, Gao et al..$^{5}$ mostraram que a utilização de probióticos no pré-operatório aumentou o número de microrganismos benéficos, proporcionando uma maior diversidade da microbiota intestinal, além de inibir potenciais agentes patogênicos. Da mesma forma, Liu et al. ${ }^{28}$ relataram que o tratamento com probióticos no pré-operatório poderia reduzir a taxa de septicemia pós-operatória e manter a barreira hepática em pacientes submetidos à cirurgia de tratamento e CCR. Recomendaram, portanto, a ingestão oral pré-operatória de probióticos, combinada com o tratamento pós-operatório em pacientes submetidos à cirurgia de ressecção de metástases hepáticas colorretais. Zhang et al. ${ }^{20}$ reforçaram que os benefícios do uso de probióticos no pré-operatório podem ser causados pelos efeitos inibitórios sobre o crescimento excessivo intestinal de E. coli, o que evita infecções e translocação bacteriana no intestino.

A radio ou quimioterapia, um dos tratamentos para CCR, causa vários efeitos colaterais, principalmente quando envolve regiões como a pelve, sendo a diarreia relatada como a principal ocorrência. Para reduzir a severidade e incidência dessa complicação, observou-se que o uso dos probióticos é seguro ${ }^{24,27}$. A utilização de probióticos antes do início da radioterapia se mostrou benéfica, pois auxiliou na prevenção da diarreia, diminuindo sua incidência e severidade induzida por toxicidade gastrointestinal provocada pela quimioterapia ${ }^{24,27}$. 
Burigo et al..$^{42}$ também relataram que a taxa de PCR foi inferior no grupo que fez uso de prebiótico pós-quimioterapia, se comparado ao grupo placebo. Isso pode indicar a ocorrência de processos inflamatórios e maior demanda metabólica, o que sugere que a quantidade de prebiótico pode ter favorecido a redução desse quadro no grupo suplementado.

\section{CONCLUSÕES}

Em resumo, os prebióticos e probióticos são eficientes coadjuvantes no tratamento do CCR porque auxiliam na diminuição da infecção pós-operatória, da incidência e severidade da diarreia e do risco de complicações pós-operatórias, como sepse, ventilação mecânica e vazamento da anastomose, além de propiciarem uma recuperação mais rápida da função intestinal. A suplementação de pro, pre e simbióticos como coadjuvantes do tratamento de CCR mostrou-se benéfica, diminuindo a severidade e incidência de complicações durante e após o tratamento, tanto cirúrgico como radio ou quimioterápico, além de proporcionar melhora na imunidade e redução do desconforto do paciente. Porém, observou-se que é importante manter a medicação pré-cirúrgica padrão. A substituição dessa medicação por apenas probióticos ou simbióticos não demonstrou efeitos benéficos no pós-operatório, sendo recomendado, portanto, o seu uso concomitante na pré-cirurgia.

Finalmente, deve-se destacar que, embora resultados positivos obtidos em trabalhos com uso de pro e prebióticos tenham sido verificados, o número limitado de artigos dificulta a generalização dos achados. Dessa forma, destaca-se a necessidade de futuros estudos de longa duração para elucidar melhor essa relação.

\section{REFERÊNCIAS}

1. Kahouli I, Malhotra M, Westfall S, Alaoui-Jamali MA, Prakash S. Design and validation of an orally administrated active $L$. fermentum-L. acidophilus probiotic formulation using colorectal cancer Apc Min/+ mouse model. Appl Microbiol Biotechnol. 2017;101(5):19992019. PMid:27837314. http://dx.doi. org/10.1007/s00253-016-7885-x.

2. Instituto Nacional de Câncer (INCA). Estimativa 2016: incidência de Câncer no Brasil. Rio de Janeiro: INCA; 2016 [citado 2017 Mar 27]. Disponivel em: http://www.inca.gov. br/estimativa/2016/tabelaestados. asp?UF=BR.

3. Brasil. Ministério da Saúde. Departamento de Informática do SUS (DATASUS). Painel de Monitoramento da Mortalidade CID-10. Brasília; 2017 [citado 2017 Mar 27]. Disponível em: http://svs.aids.gov.br/dashboard/ mortalidade/cid10.show.mtw.

4. Corrêa RS, Pinto JR. FE, Santos LV, Góis MC, Silva RP, Silva HM. Rectal cancer survival in a Brazilian Cancer Reference Unit. J Coloproctol. 2016;36:203-7.

5. Gao Z, Guo B, Gao R, Zhu Q, Wu W, Qin H. Probiotics modify human intestinal mucosa-associated microbiota in patients with colorectal cancer. Mol Med Rep.
2015;12(4):6119-27. PMid:26238090. http://dx.doi.org/10.3892/ mmr.2015.4124.

6. Oliveira A, Aarestrup FM. Avaliação nutricional e atividade inflamatória sistêmica de pacientes com câncer colorretal submetidos à suplementação com simbiótico. ABCD Arq Bras Cir Dig. 2012;25(3):147-53. PMid:23411802. http://dx.doi.org/10.1590/S010267202012000300003.

7. Chaves PL, Gorini MI. Qualidade de vida do paciente com câncer colorretal em quimioterapia ambulatorial. Rev Gaucha Enferm. 2011;32(4):767-73. PMid:22299280. http://dx.doi.org/10.1590/S198314472011000400018.

8. Theodoropoulos GE, Memos NA, Peitsidou K, Karantanos T, Spyropoulos BG, Zografos G. Synbiotics and gastrointestinal function-related quality of life after elective colorectal cancer resection. Ann Gastroenterol. 2016;29(1):56-62. PMid:26752951.

9. Machado FF, Lazzaretti RK, Paziomyck AK. Uso de prebióticos, probióticos e simbióticos nos pré e pós-operatórios do câncer colorretal: uma revisão. Rev Bras Cancerol. 2014;60:363-70.
10. Lee JY, Chu SH, Jeon JY, Lee MK, Park JH, Lee DC, et al. Effects of 12 weeks of probiotic supplementation on quality of life in colorectal cancer survivors: a double-blind, randomized, placebo-controlled trial. Dig Liver Dis. 2014;46(12):1126-32. PMid:25442120. http://dx.doi.org/10.1016/j. dld.2014.09.004.

11. Denipote FG, Trindade EB, Burini RC. Probióticos e prebióticos na atenção primária ao câncer de cólon. Arq Gastroenterol. 2010;47(1):93-8. PMid:20520982. http://dx.doi.org/10.1590/S000428032010000100016.

12. Oliveira A. O papel dos simbióticos na prevenção, tratamento e modulação da resposta inflamatória em pacientes com carcinoma colorretal. Rev Int Est Exp. 2009;1:23-31.

13. Marti del Moral AM, Moreno-Aliaga MJ, Martinez-Hernandez J. Efecto de los prebióticos sobre el metabolismo lipídico. Nutr Hosp. 2003;18(4):181-8. PMid:12884473.

14. Naspolini A. Probioticos e prebioticos e sua relação com cancer colorretal [monografia]. Criciúma: UNESC; 2012.

15. Fortes RC, Recôva VL, Melo AL, Novaes MRCG. Hábitos dietéticos de pacientes com câncer colorretal em fase pós-operatória. Rev Bras Cancerol. 2007;53:277-89. 
16. Liberali R. Metodologia científica prática: um saber-fazer competente da saúde à educação. Florianópolis: Postmix; 2008.

17. Brancher J. Uso de probióticos no tratamento da obesidade: uma revisão sistemática. Porto Alegre; 2014 [citado 2017 Mar 9]. Disponível em: http://www.lume.ufrgs.br/bitstream/ handle/10183/108988/000948985.pdf.

18. Liberati A, Altman DG, Tetzlaff J, Mulrow C, Gøtzsche PC, loannidis JP, et al. The PRISMA statement for reporting systematic reviews and meta-analyses of studies that evaluate healthcare interventions: explanation and elaboration. BMJ. 2009;339:b2700. PMid:19622552. http://dx.doi.org/10.1136/bmj.b2700.

19. Higgins JP, Green S, editors. Cochrane handbook for systematic reviews of interventions. Version 5.1.0. 2017 [citado 2017 Mar 9]. Disponível em: http://handbook.cochrane.org.

20. Zhang JW, Du P, Gao J, Yang BR, Fang WJ, Ying CM. Preoperative probiotics decrease postoperative infectious complications of colorectal cancer. Am J Med Sci. 2012;343(3):199-205. PMid:22197980. http://dx.doi. org/10.1097/MAJ.0b013e31823aace6.

21. Stephens JH, Hewett PJ. Clinical trial assessing VSL\#3 for the treatment of anterior resection syndrome. ANZ J Surg. 2012;82(6):420-7. PMid:22571474. http://dx.doi.org/10.1111/j.14452197.2012.06082.x.

22. Liu ZH, Huang MJ, Zhang XW, Wang $L$, Huang $N Q$, Peng $H$, et al. The effects of perioperative probiotic treatment on serum zonulin concentration and subsequent postoperative infectious complications after colorectal cancer surgery: a double-center and double-blind randomized clinical trial. $A m \mathrm{~J}$ Clin Nutr. 2013;97(1):117-26. PMid:23235200. http://dx.doi. org/10.3945/ajcn.112.040949.

23. Sadahiro S, Suzuki T, Tanaka A, Okada K, Kamata H, Ozaki $\mathrm{T}$, et al. Comparison between oral antibiotics and probiotics as bowel preparation for elective colon cancer surgery to prevent infection: prospective randomized trial. Surgery. 2014;155(3):493503. PMid:24524389. http://dx.doi. org/10.1016/j.surg.2013.06.002.
24. Mego M, Chovanec J, VochyanovaAndrezalova I, Konkolovsky P, Mikulova M, Reckova M, et al. Prevention of irinotecan induced diarrhea by probiotics: a randomized double blind, placebo controlled pilot study. Complement Ther Med. 2015;23(3):356-62. PMid:26051570. http://dx.doi.org/10.1016/j. ctim.2015.03.008.

25. Kotzampassi K, Stavrou G, Damoraki G, Georgitsi M, Basdanis G, Tsaousi $\mathrm{G}$, et al. A Four-Probiotics regimen reduces postoperative complications after colorectal surgery: a randomized, double-blind, placebo-controlled study. World J Surg. 2015;39(11):277683. PMid:25894405. http://dx.doi. org/10.1007/s00268-015-3071-z.

26. Kotzampassi K, Stavrou G, Damoraki G, Georgitsi M, Basdanis G, Tsaousi $\mathrm{G}$, et al. Randomized, doubleblind, placebo-controlled study of the efficacy of four probiotics to modify the risk for postoperative complications in colorectal surgery. Crit Care. 2015;19(Suppl 1):390. PMid:26549276. http://dx.doi. org/10.1186/cc14470.

27. Scartoni D, Desideri I, Giacomelli I, Di Cataldo V, Di Brina L, Mancuso $A$, et al. Nutritional supplement based on zinc, prebiotics, probiotics and vitamins to prevent radiationrelated gastrointestinal disorders. Anticancer Res. 2015;35(10):5687-92. PMid:26408744.

28. Liu Z, Li C, Huang M, Tong C, Zhang $X$, Wang $L$, et al. Positive regulatory effects of perioperative probiotic treatment on postoperative liver complications after colorectal liver metastases surgery: a doublecenter and double-blind randomized clinical trial. BMC Gastroenterol. 2015;15(1):34. PMid:25881090. http:// dx.doi.org/10.1186/s12876-0150260-z.

29. Krebs B. Prebiotic and synbiotic treatment before colorectal surgery - randomised double blind trial. Coll Antropol. 2016;40(1):35-40. PMid:27301235.

30. Yang $\mathrm{Y}, \mathrm{Xia} \mathrm{Y}$, Chen $\mathrm{H}$, Hong L, Feng J, Yang J, et al. The effect of perioperative probiotics treatment for colorectal cancer: short-term outcomes of a randomized controlled trial. Oncotarget. 2016;7(7):843240. PMid:26824990. http://dx.doi. org/10.18632/oncotarget.7045.
31. Naoum PC. Avanços tecnológicos em hematologia laboratorial. Rev Bras Hematol Hemoter. 2001;23(2):111-9. http://dx.doi.org/10.1590/S151684842001000200010 .

32. Waitzberg DL, Nardi L, Horie LM. Desnutrição em câncer. Rev Onco. 2011;2:34-7.

33. Gamallat $Y$, Meyiah A, Kuugbee ED, Hago AM, Chiwala G, Awadasseid A, et al. Lactobacillus rhamnosus induced epithelial cell apoptosis, ameliorates inflammation and prevents colon cancer development in an animal model. Biomed Pharmacother. 2016;83:536-41. PMid:27447122. http://dx.doi. org/10.1016/j.biopha.2016.07.001.

34. Reis SA, Conceição LL, Siqueira NP, Rosa DD, Silva LL, Peluzio MD. Review of the mechanisms of probiotic actions in the prevention of colorectal cancer. Nutr Res. 2017;37:1-19. PMid:28215310. http://dx.doi. org/10.1016/j.nutres.2016.11.009.

35. Mármol I, Sánchez-de-Diego C, Pradilla Dieste A, Cerrada E, Rodriguez Yoldi MJ. Colorectal Carcinoma: a general overview and future perspectives in colorectal cancer. Int J Mol Sci. 2017;18(1):E197. PMid:28106826. http://dx.doi.org/10.3390/ ijms18010197.

36. Pala V, Sieri $S$, Berrino F, Vineis $P$, Sacerdote $C$, Palli D, et al. Yogurt consumption and risk of colorectal cancer in the Italian European prospective investigation into cancer and nutrition cohort. Int $J$ Cancer. 2011;129(11):2712-9. PMid:21607947. http://dx.doi.org/10.1002/ijc.26193.

37. Sah BN, Vasiljevic T, McKechnie S, Donkor ON. Effect of probiotics on antioxidant and antimutagenic activities of crude peptide extract from yogurt. Food Chem. 2014;156:26470. PMid:24629967. http://dx.doi. org/10.1016/j.foodchem.2014.01.105.

38. Kuugbee ED, Shang X, Gamallat $Y$, Bamba D, Awadasseid A, Suliman MA, et al. Structural Change in Microbiota by a Probiotic Cocktail Enhances the Gut Barrier and Reduces Cancer via TLR2 Signaling in a Rat Model of Colon Cancer. Dig Dis Sci. 2016;61(10):290820. PMid:27384052. http://dx.doi. org/10.1007/s10620-016-4238-7. 
39. Li J, Sung CY, Lee $N, N i$, Pihlajamäki J, Panagiotou G, et al. Probiotics modulated gut microbiota suppresses hepatocellular carcinoma growth in mice. Proc Natl Acad Sci USA. 2016;113(9):E1306-15. PMid:26884164. http://dx.doi. org/10.1073/pnas.1518189113.

40. Smith PM, Howitt MR, Panikov N, Michaud M, Gallini CA, Bohlooly-Y $M$, et al. The microbial metabolites, short-chain fatty acids, regulate colonic Treg cell homeostasis. Science. 2013;341(6145):569-

73. PMid:23828891. http://dx.doi. org/10.1126/science.1241165.

41. Le Leu RK, Hu Y, Brown IL, Woodman RJ, Young GP. Synbiotic intervention of Bifidobacterium lactis and resistant starch protects against colorectal cancer development in rats. Carcinogenesis. 2010;31(2):24651. PMid:19696163. http://dx.doi. org/10.1093/carcin/bgp197.
42. Búrigo $T$, Fagundes RL, Trindade $\mathrm{EB}$, Vasconcelos $\mathrm{HC}$, Massaut IH, Rotolo MA. Ação do prebiótico sobre as proteínas de fase aguda de pacientes com neoplasia hematológica. Rev Bras Hematol Hemoter. 2007;29(2):130-5.

http://dx.doi.org/10.1590/S151684842007000200010 . 